\title{
Die Zürcher Offizin Christoph Froschauer als Wegbereiter für Johannes Honters Kosmographie (Kronstadt 1542)
}

Johannes Honter lebte im 16. Jahrhundert in Siebenbürgen und wirkte als Humanist auch auf geographisch-kartographischem Gebiet. In Kronstadt (Braşov) in Rumänien geboren, zog er im Jahre 1515 an die Wiener Universität, auf der ihn Joachim Watt (Vadianus) aus St. Gallen zur Geographie führte. Förderung auf kartographischem Gebiet verdankte er Peter Bienewitz/Apianus, der aus dem obersächsischen Leisnig stammte und später in Ingolstadt Professor der Mathematik und Eigentümer einer Buchdruckerei wurde. Seit 1525 lehrte Honter als Magister artium in der Artistenfakultät, bis ihn die drohende Belagerung Wiens durch die Türken (1529) aus der Stadt vertrieb. Gegen Ende des Jahres hielt er sich in Regensburg auf, wo sein Aufenthalt im Hause des Humanisten Johannes Turmair (Aventinus) überliefert ist. Er legte Aventin zwei während seiner Wiener Lehrtätigkeit entstandene Manu skripte einer lateinischen Grammatik und einer Kosmographie vor. Von Regensburg führte ihn das Flüchtlingsschicksal - wohl nach einem längeren Aufenthalt bei Peter Apian in Ingolstadt - nach Krakau, wo er an der Jagellonischen Universität seine Lehrtätigkeit wieder aufnehmen wollte. Da ihm dies auf die Dauer nicht gelang, wurde er in Basel Korrektor und Tutor (Verlagslektor?) in der Offizin Johann Bebel \& Michael Isengrin, bis er vom Kronstädter Rat in die Heimat zurückgerufen wurde, die seiner nach Jahren des Bürgerkrieges bedurfte.

Als Humanist war Honter von der Bildungsfähigkeit und dem Recht der Menschen auf Bildung überzeugt, wohl wissend, dass das humanistische Bildungsgut zunächst nur einem kleinen Kreis des Volkes zugutekommen könne.Während seines kurzen Krakauer Aufenthaltes liess Honter im Verlag von Matthias Scharffenberg die beiden von Wien mitgebrachten Lehrbücher erscheinen: neben der lateinischen Grammatik die Kosmographie unter dem Titel "Rudimentorum Cosmographiae libri duo. Krakau 1530". (1) Das Büchlein besteht aus 16 Blättern in Kleinoktav und ist in lateinischer Prosa abgefasst. Das erste Buch enthält die Astronomie, das zweite führt in die Geographie ein. Den beiden Teilen folgt eine Liste "Nomina locorum et gentium" mit mehrsprachigen Sachbezeichnun- gen. Das geographische Buch der Kosmographie ist ein Versuch regionalkundlicher Darstellung. Honter stellte wie einst C. Ptolemäus lange Listen topographischer Namen auf, die sich zuweilen unter Einschaltung kurzer Sätze oder weniger Worte - auf die drei alten Erdteile beziehen, während die Neue Welt mit ihrem $\mathrm{Na}-$ men Amerika unter den Inseln zu finden ist. Zur Veranschaulichung durch das Kartenbild trug Honter in der Krakauer Erstfassung nur durch einen Planiglob der Alten Welt und eine Erdkarte bei. Eine zweite Krakauer Auflage erschien unverändert im Jahre 1534. Ihr folgten in den Jahren 1534 bis 1585 Nachdrucke in der Offizin Heinrich Petri in Basel.

Nachdem sich Honter 1533 in Kronstadt niedergelassen und dort eine Buchdruckerei eingerichtet hatte, aus deren Presse seit 1539 Bücher ausgingen, brachte er seine Kosmographie in einer Neubearbeitung heraus. (2) Um das Behalten der topographischen Namen zu erleichtern, setzte er den Prosatext in Hexameter um. Diese Verfassung erweiterte den Text so beträchtlich, dass der Geographie zwei Bücher eingeräumt werden mussten. Das Titelblatt kündigte nunmehr eine Kosmographie in drei Büchern an und behielt diesen Titel auch bei, als sich die Krakauer Liste "Nomina et gentium" zu einem Abschnitt "Cum vocabulis rerum" erweiterte, der im Textteil als viertes Buch geführt, auf dem Titelblatt jedoch gesondert angezeigt wur de. Nach der vorläufigen Ausgabe Kronstadt 1541 und einer Zwischenausgabe Breslau 1542 erschien die endgültige Ausgabe unter dem Titel "Rudimenta Cosmographica. Kronstadt 1542" ohne Nennung des Verfassers. (3) Im Textteil bedingte die Versfassung eine starke Beschränkung des topographischen Namengutes. Von den 1400 Namen der Krakauer Erstfassung fielen gegen 500 Namen aus. Ergänzt wurde der Text diesmal durch $16 \mathrm{Tafeln}$, von denen die ersten drei Zeichnungen für das astronomische Buch enthalten, während die übrigen dreizehn einen Atlas minor mit Erdkarte und zwölf Länderkar ten bilden. Die Erdkarte "Universalis Cosmographica" trägt Honters Monogramm IHC = Johannes Honterus Coronensis und die Verlags-

Dr.G.Engelmann, Paul - Neumann - Strasse 85, DDR 1502 Potsdam-Babelsberg. 
angabe Corona M. D. XLII. Den Schnitt der Holzstöcke für die 16 Tafeln besorgte Honter mit eigener Hand. $\mathrm{Zu}$ seinen Lebzeiten erschien noch eine Ausgabe im Jahre 1548 oder 1549. Den Abschluss der Originalausgaben bildete die postume Ausgabe des Jahres 1577 oder 1578 . In der Zwischenzeit setzten die Nachdrucke der Kronstädter Neufassung der Kosmographie Honters ein. Um sie bemühte sich am meisten die Offizin Christoph Froschauer in Zürich.Anfang des Jahres 1544 übersandte Martin Hentius, der nach Abschluss seines Wittenberger Stu diums nach Kronstadt zurückgekehrt war, an Bullinger, den Nachfolger Ulrich Zwinglis in Zürich, ein Angebinde (munusculum), (4) unter dem Honters Kosmographie in der Kronstädter Ausgabe von 1542 vermutet wird. (5) Dieses Angebinde gab Bullinger an seinen Gevatter Christoph Froschauer d. Ae., dem das Büchlein für einen Nachdruck willkommen war. Im Sommer 1546 übersandte dann Froschauer an Joachim Vadian ein "nüws büchli":"Ist erst der stund ussgangen, und ist das exemplar uss Sybenbürgen meister Heinrich Bullinger zugeschickt. "(6) Dieses neue Büchlein ist Froschauers erster Nachdruck von 1546, frisch aus der Presse gekommen. Froschauer übernahm Honters Text der Kronstädter Ausgabe wortgetreu und sorgte durch seine Korrektoren für einen fehlerfreien Satz. So ist es verständlich, wenn die siebenbürgisch-sächsische Forschung die Kosmographie von Honters eigener Kronstädter Ausgabe (1542) bis zum letzten Zürcher Nachdruck in der Offizin Froschauer (1602)

als ein einheitliches Werk ansah. (7)

Von den sechzehn Zürcher Ausgaben der Offizin Froschauer, deren Drucksignet ein Weidenbaum mit fünf Fröschen war, entfielen je sieben Ausgaben auf Christoph Froschauer d. Ae. und auf dessen Neffen Christoph Froschauer d. J. einschliesslich der Brüder Escher als Nachfolger im Verlag, wobei sie als ungelernte Drucker den Betrieb mit dem alten Personal weiterführten. Als 1591 Johannes Wolff die Offizin übernommen hatte, erschienen noch zwei Ausgaben der Zürcher Nachdrucke.

Christoph d. Ae. liess die ersten beiden Ausgaben Zürich 1546 und 1548 (a) anonym unter dem Titel "Rudimenta Cosmographica" auf den Markt bringen. Seine folgenden Ausgaben Zü- rich 1548 (b), 1549, 1552, 1558 und 1564 tragen den Titel "Rudimentorum Cosmographicorum Johannis Honteri Coronensis libri tres, cum tabellis geographicis elegantissimis", wobei auf das vierte Buch der Nomenklatur mit den Worten verwiesen wird: "De variarum rerum nomenclatoris per classes, liber unus". Unter Christoph d. J. und den Brüdern Escher erschienen unverändert die Ausgaben Zürich 1565, 1570, $1573,1581,1583$ und 1590. Johannes Wolff wählte für die letzten beiden Ausgaben Zürich 1597 und 1602 den Titel: "Enchiridion Cosmographiae - - auctore Johanne Hontero Coronense." Schwieriger als die Uebernahme des Textes war der Nachdruck von Honters Atlas minor. Christoph Froschauer d. Ae. liess die 16 Tafeln des Anhangs mit der Erdkarte und den 12 Länderkarten neu zeichnen und in Holz schneiden: "hab die figuren mit grossen Kosten lassen machen." (8) Seine Werkstatt für den Holztafeldruck unterstand während der Jahre 1544 bis 1546 Heinrich Vogtherr d. Ae., einem Maler und Formschneider aus Dillingen a.d. Donau. Er kam 1544 zu Froschauer nach Zürich, um bei der Illustration der Schweizerchronik des Johannes Stumpf mitzuwirken. Zusätzlich über nahm er den Neuschnitt der 13 Honterkarten. Auf die Erdkarte setzte er unter Wegfall von Honters Monogramm sein eigenes $\mathrm{HVE}=\mathrm{Hein}-$ rich Vogtherr der Aeltere und die Verlagsangabe Tiguri 1546, womit er das Jahr der ersten Ausgabe der Nachdrucke Froschauers festhielt. Johannes Wolff änderte dieses Verlagsjahr in den letzten beiden Nachdrucken in "1596" ab, um für den seit 1546 unveränderten Karteninhalt eine jüngste Auflage vortäuschen zu können. Eine weitere Jahreszahl, die Vogtherr 1546 einsetzte, verwirrte die Chronologie der Kartenfolge. Bei der Neuzeichnung der astronomischen Tafel I schnitt er in die Randbordüre die Jahreszahl "1530" in Spiegelschrift ein. Vermutlich wollte er in einer absichtlich ungewöhnlichen Form die Erinnerung an Honters Krakauer Erstausgabe von 1530 festhalten. Ausserdem übernahm die Offizin Froschauer in zwei weitere Verlagswerke Honters Atlas minor bzw. Karten aus demselben: in Joachim Vadians "Epitome trium terrae partium" (Zürich $1548 \mathrm{mit}$ Neuauflagen bis nach 1564) und in die Schweizerchronik Stumpfs (Zürich 1548 bis 1606). - Auf 
den Länderkarten des Atlas minor liess Froschauer, um die Betriebskosten zu senken, die topographischen Namen, die Honter ebenfalls in die Druckstöcke geschnitten hatte, in Buchdrucklettern setzen und die Metallzeilen in Vertiefungen des Holzstockes versenken. Dabei entstand bei unachtsamer Verwendung der Druckstöcke die Gefahr, dass einzelne Namen teilweise oder ganz ausfielen. Beim Wiedereinsetzen war das Einfügen ausgefallener Namen an einem falschen Ort bedenklich. (9) So konnten Froschauers Nachdrucke an Honters kartographisches Meisterwerk nicht herankommen.

Wie kräftig die Offizin Christoph Froschauer zur Verbreitung von Honters Kosmographie beitrug, geht aus der Uebersicht über ausgewählte Bibliotheken hervor, die Honters Kosmographie in den Originalausgaben Honters wie Froschauers Nachdrucken besitzen (s. Liste!). Während Honters Krakauer und Kronstädter Ausgaben von den Bibliotheken in Kronstadt und Hermannstadt, Krakau und Warschau, Budapest und Wien (ausserdem Paris) nur in einzelnen Ausgaben angeschafft wurden, setzte mit Froschauers Nachdrucken ein weltweites Interesse an Honters Kosmographie ein. Die erste Zürcher Nachdruckausgabe von 1546 nahmen von den 16 ausgewählten Bibliotheken sieben in ihre Buchbestände auf: Berlin, Budapest, Dresden, London, Moskau, Wien und Zürich. (10) Die Nationalbibliothek "Széchéyni" Budapest besitzt sämtliche Zürcher Ausgaben von 1546 bis 1590; ihr fehlen nur die beiden letzten Ausgaben, die Froschauers Nachfolger Johannes Wolff herausbrachte. Dann folgen mit 7 Ausgaben Zürich und Dresden, mit 6 Ausgaben Hermannstadt und Berlin (das Kriegsverluste erlitt), mit 5 Ausgaben Klausenburg, Wien, Prag und London, mit 4 Ausgaben Krakau, Paris und Washington usw. So trug die Offizin Christoph Froschauer von Zürich aus Honters Namen als den eines Geographen und Kartographen bis ins 17. Jahrhundert durch Deutschland und darüber hinaus. Antonius Verantius, der dalmatinische Prälat und Probst des Siebenbürgischen Kapitels, der spätere Bischof von Erlau und Erzbischof in Gran rühmte 1549, durch die Verbreitung von Honters Kosmographie sei Siebenbürgen bekannter und Kronstadt berühmter geworden. (11)
Fussnoten:

(1)G. Engelmann: Die Kosmographie des Johannes Honter in ihrer Krakauer Erstfassung 1530. In: Studia z dziejów geografii i kartografii. Etudes d'histoire de la Géographie et de la Cartographie sous la rédaction de Józef Babicz. In:Polska Akademia nauk. Zakad historii nauki i techniki Monografie z dziefów nauk i techniki, tom LXXXVII. Wrocaw, Warszawa, Kraków, Gdańsk 1973, S. 319-333.

(2) G. Engelmann: Johannes Honter als Kartograph (im Druck).

(3) O. Netoliczka: Johannes Honterus' ausgewählte Schriften. Wien \& Hermannstadt 1898, S. 153-196.

(4) Brief Martin Hentius an Heinrich Bullinger, Kronstadt 1544 I 3. In: K. Reinerth:Martinus Hentius aus Kronstadt über den Lehrunterschied zwischen Wittenberg und der Schweiz in der Abendmahlsfrage im Jahre 1543. In:Archiv für Reformationsgeschichte. Gütersloh Bd. 54. 1963, S. $181-198$ (189).

(5) Als erster vermutete dies $1908 \mathrm{E}$. Arbenz, der Bearbeiter der Vadianischen Briefsammlung der Stadtbibliothek St. Gallen. Bd. VI, 2, hrsg. von E. Arbenz u. H. Wartmann. In: Mitteilungen zur Vaterländischen Geschichte. St. Gallen, Bd. 30, 2 (III. Folge Bd. 10) 1908, S. 560, Nr. 1491, Anm. 2 .

(6) Brief Christoph Froschauer an Joachim Vadian, Zürich 1546 VIII 20. In:Ebenda 1908, S. $560, \mathrm{Nr} .1491$.

(7) F. Teutsch benutzte z. B. bei der metrischen Uebertragung des Hexametertextes ins Deutsche Froschauers zweiten Nachdruck Zürich 1548. Siehe F. Teutsch:Drei sächsische Geographen des sechzehnten Jahrhunderts. In: Archiv des Vereins für siebenbürgis che Landeskunde. Hermannstadt. Neue Folge, Bd. 15. 1880, S. 590 ff.

(8) Brief Christoph Froschauer an Joachim Vadian, Zürich 1546 VIII 20. In: a. a. O. 1908. S. 560, Nr. 1491.

(9) G. Engelmann: Topographische Namen auf Holzschnittkarten des 16. Jahrhunderts. In:Karten in Bibliotheken (Festschrift Heinrich Kramm 1971). In:Kartensammlung und Kartendokumentation, Bd. 9. Bonn-Bad Godesberg 1971, S. 33-50. (10) Die Angaben für die Liste: Standorte der Ausgaben in grossen Bibliotheken wurden den Beständen bzw. Katalogen folgender Bibliotheken entnommen: 
Berlin: Deutsche Staatsbibliothek Berlin(DDR) Staatsbibliothek Preussischer Kultur besitz Berlin (West)

Budapest: Nationalbibliothek "Széchényi"Budapest

Bukarest: Bibliotheca Academia R. S. România Bucaresti

Dresden: Sächsische Landesbibliothek Dresden

Hermannstadt: Muzeul Brukenthal, Bibliotecă, Sibiu

Klausenburg: Bibliotheca Academia R. S. Romania, Filiala Cluj

Krakau: Biblioteka Jagiellonska Krakow

Kronstadt: Dokumentarbibliothek der evang. Honterusgemeinde Braşov
London: British Museum, Map Room, London Moskau: Lenin-Bibliothek Moskau Paris: Bibliotheque Nationale Paris Prag: Stātni Knihovna Praha Warschau: Biblioteka Narodowa Warszawa Washington: Library of Congress Washington Wien: Oesterreichische Nationalbibliothek Wien Zürich: Zentralbibliothek Zürich

(11) Brief Anton Verantius an Christian Pomarius, Weissenburg 1549 Vii 15. In: Antonius Verantius (Anton Wrančič): Sämtliche Werke, hrsg. von L. Szalay. Bd. VI: Antonii Verancii Epistolae. In: Monumenta Hungariae Historica, Abt. II: Scriptores, Bd. 9. Budapest 1860, S. $329-333, \mathrm{Nr} .140$.

\begin{tabular}{|c|c|c|c|c|c|c|c|c|c|c|c|c|c|c|c|c|c|c|c|c|c|c|c|c|}
\hline \multicolumn{25}{|c|}{ Standorte der Originalausgaben Honters und Froschauers Nachdrucken in Bibliotheken } \\
\hline \multirow[b]{2}{*}{$\begin{array}{l}\text { Standorte } \\
\text { grosser } \\
\text { Bibliotheken }\end{array}$} & \multicolumn{7}{|c|}{$\begin{array}{l}\text { Johannes Honter } \\
\text { Krakau Kronstadt }\end{array}$} & \multicolumn{16}{|c|}{ Christoph Froschauer } & \\
\hline & $\begin{array}{l}0 \\
\text { in } \\
\text { in } \\
\end{array}$ & $\begin{array}{l}H \\
\text { in } \\
-1\end{array}$ & 站 & \begin{tabular}{l}
$*$ \\
\multirow{2}{*}{} \\
$\stackrel{\sim}{*}$
\end{tabular} & $\underset{\substack{+-1}}{N}$ & 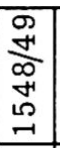 & 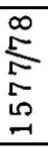 & $\begin{array}{l}0 \\
\text { L } \\
\\
-1 \\
\end{array}$ & 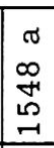 & 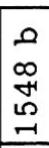 & 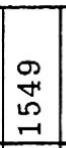 & 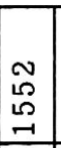 & 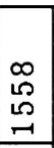 & $\begin{array}{l}4 \\
0 \\
0 \\
\\
\end{array}$ & 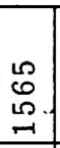 & 온 & 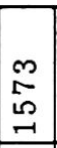 & \begin{tabular}{c}
$\infty$ \\
\multirow{2}{\infty}{} \\
$\sim$ \\
\end{tabular} & $\begin{array}{l}-1 \\
\infty \\
10 \\
-1 \\
\end{array}$ & $\begin{array}{l}m \\
\infty \\
\infty \\
\sim \\
\sim\end{array}$ & $\begin{array}{l}\circ \\
\stackrel{\circ}{\circ} \\
\stackrel{1}{-1} \\
\end{array}$ & $\begin{array}{l}5 \\
0 \\
0 \\
-1 \\
\end{array}$ & $\begin{array}{l} \\
\text { O } \\
0 \\
-1 \\
\end{array}$ & \\
\hline $\begin{array}{cc}\text { Berlin } & \text { DSB } \\
\text { SBPK }\end{array}$ & & & & & & & & $\bullet$ & & & - & - & $\bullet$ & $\bullet$ & $\bullet$ & & & & & & & & & $6\left\{\begin{array}{l}2 \\
4\end{array}\right.$ \\
\hline Budapest & & $\bullet$ & & • & • & • & & • & $\bullet$ & - & $\bullet$ & • & $\bullet$ & $\bullet$ & $\bullet$ & - & $\bullet$ & $\bullet$ & $\bullet$ & $\bullet$ & $\bullet$ & & & 18 \\
\hline Bukarest & & & & & & & & & & & & $\bullet$ & & & & & - & & & & & & & 2 \\
\hline Dresden & & & & & & & & $\bullet$ & $\bullet$ & $\bullet$ & $\bullet$ & $\bullet$ & & $\bullet$ & & & & & & $\bullet$ & & & & 7 \\
\hline Hermannstadt & & & - & & $\bullet$ & & & & $\bullet$ & & & - & & & $\bullet$ & & & & & - & $\bullet$ & - & & 8 \\
\hline Klausenburg & & & & & & & & & $\bullet$ & & & - & $\bullet$ & & $\bullet$ & & & & & & & $\cdot$ & - & 5 \\
\hline Krakau & $\bullet$ & $\bullet$ & - & & & & & & & $\bullet$ & $\bullet$ & $\bullet$ & & & & & $\bullet$ & & & & & & & 7 \\
\hline Kronstadt & & & $\bullet$ & & - & & - & & & & - & & $\bullet$ & & & & & & & & & & - & 6 \\
\hline London & & & & & & & & $\bullet$ & & - & & & - & & & & $\bullet$ & & & $\bullet$ & & & & 5 \\
\hline Moskau & & & & & & & & $\bullet$ & & & & & & & & & & & & $\bullet$ & & & & 2 \\
\hline Paris & & $\bullet$ & & & & & & & & & $\bullet$ & $\bullet$ & & & & & & & & $\bullet$ & & $\bullet$ & & 5 \\
\hline Prag & & & & & & & & & & $\bullet$ & $\bullet$ & $\bullet$ & & $\bullet$ & & $\bullet$ & - & & & & & & & 5 \\
\hline Warschau & - & & & & & & & & & & & & & & & & & & & & & & & 1 \\
\hline W ashington & & & & & & & & & & $\bullet$ & $\bullet$ & & & $\bullet$ & & & & & $\bullet$ & & & & & 4 \\
\hline Wien & & & & & - & & & $\bullet$ & $\bullet$ & & - & & & & & $\bullet$ & • & & & & & & & 6 \\
\hline Zürich & & & & & & & & $\bullet$ & & $\bullet$ & - & - & & & & - & $\bullet$ & & & - & & & & 7 \\
\hline & 2 & 3 & 3 & 1 & 4 & 1 & 1 & 7 & 5 & 7 & 10 & 10 & 5 & 5 & 4 & 4 & 6 & 1 & 2 & 7 & 2 & 2 & 2 & 94 \\
\hline
\end{tabular}

(*Breslau) 